\title{
A clinical study of anterior segment applications of neodymium-doped: yttrium aluminium garnet laser
}

\begin{abstract}
Background: $\mathrm{Nd}$ :YAG laser finds a number of applications in the treatment of anterior segment diseases like primary angle closure and postoperative complications of extracapsular cataract surgery.

Aim: To assess the safety and efficacy of Nd:YAG laser in the management of diseases of anterior segment.

Methods: This prospective study included patients who underwent Nd:YAG laser therapy for the treatment of anterior segment diseases during a one year period. The nature of procedure, complications during and after laser treatment and outcomes were noted. Patients were followed upto 3 months post treatment.

Results: 103 eyes of 75 patients were included. The age of patients ranged from 41 72 years (42 males and 33 females). Nd:YAG laser posterior capsulotomy $(n=38)$ was the most common indication followed by laser peripheral iridotomy $(n=30)$, anterior vitreolysis(n=4), Fibrin membrane lysis $(n=2)$ and corticolysis $(n=1)$. There was an improvement in visual acuity from baseline in patients with posterior capsular opacification and cystoid macular edema due to vitreous-tug syndrome. There was a reduction in the IOP from baseline and widening of the anterior chamber angles following peripheral iridotomy in $89 \%$ of the patients with primary angle closure disease. Hyphaema and transient IOP elevation were the most common complications encountered which resolved with therapy.

Conclusion: Nd:YAG laser is a safe and effective means of treating the spectrum of primary angle closure disease and complications of extracapsular cataract surgery. It has a better acceptance by patients, is cost-effective, may be performed as an outpatient procedure and avoids complications of surgery.
\end{abstract}

Volume 8 Issue 2 - 2018

\author{
Anitha S Maiya,' Pranessh Ravi ${ }^{2}$ \\ 'Department of Ophthalmology, J J M Medical College, India \\ ${ }^{2}$ Adichunchanagiri institute of medical sciences and research, \\ India
}

Correspondence: Pranessh Ravi, Junior resident, Department of ophthalmology, Adichunchanagiri institute of medical sciences and research, B G Nagara - 57|448, Karnataka, India Tel +9l-9686955322; Email pravianth@gmail.com

Received: February 21, 2018| Published: March 07, 2018

\section{Introduction}

The neodymium:yttrium-aluminum-garnet (Nd:YAG) laser is a solid-state laser having a wavelength of $1064 \mathrm{~nm}$. It causes photodisruption of the ocular tissues by with a short, high-power pulse. Optical breakdown results in ionization, or plasma formation, in the ocular tissue which produces acoustic and shock waves that disrupt the target tissue. Nd:YAG laser finds a number of applications in the various anterior segment diseases. The most important anterior segment application of Nd:YAG laser is perhaps in the management of primary angle closure disease. Nd:YAG laser iridotomy to relieve pupillary block is safe and effective in treating or preventing angleclosure glaucoma. ${ }^{1,2}$ It can also be used to effectively treat a number of disorders arising as a complication of extracapsular cataract surgery. The most common being the posterior capsular opacification which can occur in almost $50 \%$ of the patients within 3-5 years of surgery. The other complications like pupillary block glaucoma, inflammatory fibrinous membrane covering the pupil, vitreous-tug syndrome, localized posterior synechiae, inflammatory precipitates on the intraocular lens, anterior capsular phimosis can be effectively managed by using the photo disruptive effects of Nd:YAG laser. The advantages of Nd:YAG laser is that it is a quick procedure performed in a closed eye without the risks of surgical entry into the eye there by reducing the complications such as infection. It has a better acceptance by patients and may be done as an outpatient procedure under topical anaesthesia, and is cost-effective. This study was conducted to determine the various anterior segment applications of Nd:YAG laser among our patients.

\section{Methods}

This prospective study included all the consecutive patients who underwent Nd:YAG laser therapy for various indications during a one year period. Approval of the Institutional Ethics committee was obtained. The records of all the patients were reviewed to note the demographic data, indications for the Nd:YAG laser therapy and nature of the laser procedure. The laterality of the procedure, energy level, total number of shots, total energy used, complications occurring during and after the procedure, post-laser treatment prescribed were noted. The intended outcomes of the procedure (like a patent peripheral iridotomy with relieving of pupillary block, improvement in the best corrected visual acuity after posterior capsulotomy etc) were analysed. All the patients underwent a comprehensive ophthalmic evaluation including the assessment of best corrected visual acuity, intraocular pressure measurement, slit-lamp examination, gonioscopy and posterior segment evaluation by direct and indirect ophthalmoscopy.

The Nd:YAG laser used was the $1064 \mathrm{~nm}$ laser from Carl Zeiss Meditech. A written informed consent had been obtained from all patients prior to the procedure. The appropriate laser procedure was done by a single consultant (ASM) using standardized protocols. All patients were treated with a single application of Brimonidine eye drops one hour prior to the laser procedure and one drop immediately 
after the procedure and IOP was measured one hour after the procedure. The post laser therapy included topical steroids (dexamethasone $\%$ QID for 5 days) and oral Acetazolamide (250mg BD/TID for 3 days). All the patients were followed up one week after the procedure and the visual acuity, complications and achievement of the desired outcome was recorded. Repeat procedures if any (like enlargement of the peripheral iridotomy/posterior capsulotomy) was noted.

\section{Results}

A total of 75 patients (103 eyes) underwent Nd:YAG laser procedures during the one year study period. The details of the procedures done are as given in Table 1. Nd:YAG laser peripheral iridotomy: Laser peripheral iridotomy was done in 56 eyes of 30 patients. The patients belonged to the age group of 40-69 years. 12 patients were males and 18 patients were females. The main indication was primary angle closure disease Nd:YAG laser posterior capsulotomy: Posterior capsular opacification was seen in 38 patients. Nd:YAG laser Posterior capsulotomy was performed 40 eyes of 38 patients aged between 58-72 years. The duration between the cataract surgery and posterior capsulotomy ranged between 8 months to 6 years. Majority of the patients ( 35 eyes) had undergone small incision cataract surgery; phacoemulsification had been done in only 5 eyes. Type of PCO was Elshnig's pearl type in 27 eyes and fibrous type in 13 eyes. The energy used for the capsulotomy ranged between 1.0-2.4 $\mathrm{mJ}$; average number of shots was 40 and both the energy level and number of shots depended on the thickness of the PCO. The pattern of laser capsulotomy done was cruciate type in 36 eyes and 'Christmastree' type in 4 eyes (Table 2) (Table 3).

Table I Nd:YAG laser procedures performed

\begin{tabular}{lll}
\hline $\begin{array}{l}\text { Nd:YAG laser } \\
\text { procedure }\end{array}$ & $\begin{array}{l}\text { Number of } \\
\text { patients }\end{array}$ & Number of eyes \\
\hline Peripheral iridotomy & 30 & 56 \\
Posterior capsulotomy & 38 & 40 \\
Anterior vitreolysis & 4 & 4 \\
Fibrin membrane lysis & 2 & 2 \\
Laser corticolysis & $\mathrm{I}$ & $\mathrm{I}$ \\
Total & 75 & 103 \\
\hline
\end{tabular}

Table 2 Indications for laser Peripheral iridotomy in our patients

\begin{tabular}{lll}
\hline Indications & $\begin{array}{l}\text { Number of } \\
\text { patients }\end{array}$ & $\begin{array}{l}\text { Number } \\
\text { of eyes }\end{array}$ \\
\hline PACS* & 23 & 46 \\
PAC\# & 2 & 4 \\
PACG+ & I & 2 \\
$\begin{array}{l}\text { Pseudoexfoliation syndrome with } \\
\text { secondary angle closure }\end{array}$ & 2 & 2 \\
$\begin{array}{l}\text { Traumatic secondary angle closure } \\
\text { Post-cataract surgery secondary angle } \\
\text { closure }\end{array}$ & I & I \\
TOTAL & 30 & I \\
\hline
\end{tabular}

\section{Nd:YAG laser anterior segment vitreolysis}

We encountered 4 eyes of 4 patients with 'vitreous-tug syndrome' who underwent laser vitreolysis. All of them had undergone manual small incision cataract surgery with intra-operative rupture of the posterior capsule and vitreous loss during the surgery. Three of these patients had a sulcus placed posterior chamber PMMA intraocular lens and one patient was aphakic. One patient had recurrent cystoid macular edema; two patients had pupillary distortion with glare and in one patient it was done as a prophylactic measure. Miosis of the pupil by $2 \%$ pilocarpine nitrate eye drops was done in all patients one hour prior to the procedure. The energy used was between 0.5 $1.5 \mathrm{~mJ}$ and the laser spots were applied close to the cataract wound using an Abraham lens to help in good visualization. It was possible to completely lyse the vitreous strand using the laser with approximately $15-20$ shots.

Table 3 Visual outcome of Nd:YAG laser posterior capsulotomy

\begin{tabular}{lll}
\hline BCVA & $\begin{array}{l}\text { Before laser } \\
\text { (eyes) }\end{array}$ & $\begin{array}{l}\text { After laser } \\
\text { (eyes) }\end{array}$ \\
\hline $6 / 9-6 / 6$ & - & 28 \\
$6 / 18-6 / 12$ & 4 & 10 \\
$6 / 60-6 / 24$ & 28 & 2 \\
$<6 / 60$ & 8 & - \\
TOTAL & 40 & 40 \\
\hline
\end{tabular}

\section{Nd:YAG laser membranectomy}

Postcataract surgery inflammatory membrane over the pupil was seen in 2 eyes of 2 patients with severe postoperative uveitis within 10 days of surgery. In both the patients a laser membranectomy was done using energy between $0.5-2 \mathrm{~mJ}$ with the laser spots applied close to the pupillary margin causing the membrane to separate and the pupil to dilate. Both the patients were started on intensive topical steroid drops and cycloplegics which were tapered based on the clinical response.

\section{Nd:YAG laser corticolysis}

$\mathrm{Nd}$ :YAG laser corticolysis was performed in one patient who had residual cortex after manual small incision cataract surgery. Using the energy settings of 3.4-4.0 $\mathrm{mJ}$ laser was focused behind the posterior chamber intraocular lens within the substance of the cortical matter without disrupting the posterior capsule. The patient received intensive topical steroid therapy. At the 4 weeks follow up, the residual cortical matter had reduced causing an improvement in the best corrected visual acuity from $4 / 60$ before laser to $6 / 18$.

\section{Discussion}

Nd:YAG laser finds numerous applications in the treatment of anterior segment pathologies. It has provided an option for closedeye, effective, and relatively safe technique of management of several commonly encountered anterior segment diseases entities.

\section{Nd:YAGlaser peripheral iridotomy}

The energy used for the iridotomy ranged between $2.8-5.0 \mathrm{~mJ}$ and depended on the colour and thickness of the iris with thicker and darkly pigmented irides requiring higher energy levels. The average number of shots required was about 12 . The peripheral iridotomy was performed in the superotemporal quadrant in 48 eyes, superonasal quadrant in 2 eyes and inferotemporal quadrant in 6 eyes. Only $50 \%$ of our patients reported a mild ocular discomfort/pain during the procedure; the remaining patients were comfortable during the procedure. Enlargement of the previously performed iridotomy was required in 2 eyes. The complications encountered during the 
procedure was bleeding from the iris leading to mild hyphaema in 5 eyes and corneal endothelial burn in 2 eyes. Both these complications resolved without any sequelae. None of our patients developed a post laser IOP spike. A mild grade of iritis was seen at one week follow up visit in 2 eyes which recovered with a short course of topical steroids.

It was observed that in $89 \%$ of the eyes (50 eyes), the angles were widened quantitatively as seen on gonioscopy after laser iridotomy (median angle width increased by two Shaeffer grades). There was a reduction in the IOP from baseline values after laser iridotomy. 3 patients ( 6 eyes) with peripheral anterior synechiae of more than 180 degrees required medical therapy to control IOP even after laser and one patient underwent cataract extraction with trabeculectomy and one patient underwent cataract extraction with implantation of posterior chamber intraocular lens. Our findings are consistent with the study by Nolan and associates who found that Nd:YAG laser iridotomy is an effective method of widening the drainage angle and reducing the IOP in patients with primary angle closure. ${ }^{3}$

\section{Nd:YAG laser posterior capsulotomy}

Posterior capsular opacification (PCO) is the most common long term complication of cataract surgery ${ }^{4}$ which leads to a reduction in the BCVA and distortion of images. Nd:YAG laser posterior capsulotomy was performed in 40 eyes of 38 patients. In our study 36 patients $(90 \%)$ attained a best corrected visual acuity of $6 / 12$ or better which is comparable with the study by Bhargava et al. ${ }^{5}$ who found that about $94.6 \%$ of patients attained a best corrected visual acuity of $6 / 12$ or better after Nd:YAG laser posterior capsulotomy. The complications noted during the procedure was pitting of the intraocular lens in 2 eyes which was trivial and visually insignificant. A secondary increase in IOP was seen in 2 eyes at 1 week follow up which was transient and resolved with medical therapy.

\section{Nd:YAG laser anterior segment vitreolysis}

Incarceration of vitreous strand in the cataract surgery incision can lead to complications like cystoid macular edema, edge glare/ photophobia, distortion of the pupil/corectopia, keratopathy and rarely retinal detachment (as a result of vitreous traction in the peripheral retina). Nd:YAG laser may be considered as a method of choice for initial treatment of vitreous strands in the surgical wounds as reported in the study by Levy and Pisacano ${ }^{6} \mathrm{Nd}$ :YAG laser vitreolysis was performed in 4 eyes of 4 patients with 'vitreous-tug syndrome'. There were no postlaser complications in any of the 4 eyes until one month after the procedure. There was an improvement in the best correct visual acuity in the patient with the CME from 6/36 to 6/12.

\section{Nd:YAG laser membranectomy}

Postcataract surgery inflammatory membrane may be encountered in patients with severe postoperative uveitis and may lead to pupillary block secondary angle closure glaucoma if not treated. Nd:YAG laser was found to be successful in lysing postoperative pupillary membranes in a series of 7 patients with clearing of the zone of visual axis and improvement in vision. ${ }^{7}$ In our patients, laser membranectomy successfully lysed the inflammatory membrane and the postoperative uveitis resolved completely with topical steroids and cycloplegics in both the patients with improvement in visual acuity by 4 weeks follow up visit. One patient developed elevated intraocular pressure post-laser which resolved with medical therapy.

\section{Nd:YAG laser corticolysis}

$\mathrm{Nd}$ :YAG laser corticolysis is the treatment of residual cortical matter after extracapsular cataract extraction to accelerate the process of absorption of the cortical matter or to prevent the formation of a thick membrane. It has been reported that Nd:YAG laser can be used to lyse residual cortex after cataract surgery resulting in an improvement of visual acuity. ${ }^{8}$ We attempted this procedure in 1 patient and found a reduction in the amount of cortical matter and improvement in the best corrected visual acuity at 4 weeks post laser. However, a study with larger number of subjects would be needed to validate the findings. Aspiration of the residual cortex may be a better option since it can completely remove the residual cortex.

\section{Conclusion}

$\mathrm{Nd}$ :YAG laser finds many applications in the treatment of anterior segment ocular diseases. It is a safe and effective technique with very few treatable complications if performed with care taking appropriate precautions. In many conditions like primary angle closure disease and posterior capsular opacification it has replaced surgical interventional procedures and hence is more comfortable for patients with early recovery.

\section{Acknowledgments}

None.

\section{Conflicts of interest}

None.

\section{References}

1. American Academy of Ophthalmology. Laser peripheral iri-dotomy for pupillary-block glaucoma. Ophthalmology. 1994;101(10):1749-1758.

2. Fleck BW, Dhillon B, Khanna V, et al. A randomised, pro-spective comparison of Nd:YAG laser iridotomy and opera-tive peripheral iridectomy in fellow eyes. Eye. 1991;5(Pt 3):315-321.

3. Nolan WP, Foster PJ, Devereux JG, et al. YAG laser iridotomy treatment for primary angle closure in east Asian eyes. $\mathrm{Br} J$ Ophthalmol.2000;84(11):1255-1259.

4. Bhargava R. A review of Posterior capsule opacification. J Ophthalmic Pathol. 2014;3(4).

5. Bhargava R, Kumar P, Prakash A, et al. Estimation of mean Nd:YAG laser capsulotomy energy levels for membranous and fibrous posterior capsule opacification. Nepal J ophthalmol.2012;4(7):108-113.

6. Levy JH, Pisacano AM. Clinical experience with Nd:YAG laser vitreolysis in the anterior segment. J Cataract Refract Surg. 1987;13(5):548-550.

7. Gandham SB, brown RH, KatzLJ, etal. Neodymium:YAGmembranectomy for pupillary membranes on posterior chamber intraocular lenses. Ophthalmology. 1995;102(12):1846-1852.

8. Hood CT, Shtein RM, Mian SI, et al. Neodymium-yttrium-aluminiumgarnet laser lysis of retained cortex after phacoemulsification cataract surgery. Am J Ophthalmol. 2012;154(5):808-813. 